\title{
ONE ASYMPTOTIG PROPERTY OF BEHAVIORAL CHANGE IN TERMS OF PROBABILITY DISTRIBUTION
}

\author{
IN-MAO LIU
}

National Taiwan University

If there exists some general response tendency which can be related to all known basic behavioral measures in clear-cut equations, the theoretical structure thus constructed will be elegant. Hull (1943) attempted to construct such a structure by means of the concept reaction potential. At the same time he recognized the difficulty. For instance, in discussing the relation of ${ }_{s} t_{r}$ to $E$, learning situation is restricted to one in which the promptness of the reward is dependent upon the promptness of the response. However in his formal postulate set relating the behavior tendency to four behavioral indices, there are no such restrictions throughout in his " Principles of Behavior". Thus, the probability of reaction evocation undergoes a change in an ogival relationship to effective reaction potential, reaction latency stands in a negatively accelerated inverse relationship and both resistance to experimental extinction and reaction amplitude are increasing linear functions of $E$. This postulate set is consistent with the introduction of reaction potential, a generalized response tendency. As we have mentioned, Hull came across difficulty with respect to the consequences of postulating $E$. For, under certain experimental condition of reward administration, each response index does not follow the direction of change as stated. Specifically, amplitude measure will take the expected direction of change, if a rat can be given more food the harder it presses a bar in a Skinner box. Whereas in case the amount of food given for each press of the bar is constant, the force applied to the bar by a rat will not take the predicted change.

Spence (1956) employed two different equations for determining reaction potential depending on whether an experimenter uses the classical or instrumental conditioning situation. Nevertheless, the question remains. With which response measure is the determined reaction potential to be associated? If the relation formula connecting $E$ and one of response measures depends on a particular situation, then the elegance of the theoretical structure attributed to the postulation of $E$ does no longer exist.

Logan's micromolar approach (Logan, 1956) arises from the consideration of the instrumental conditioning in which the conditions of reward can be manipulated independently of performance. The basic notion of micromolar theory is that responses that differ quantitatively are considered to be different responses. However, introduction of the new terminology will be unnecessary if one considers the quantitatively different responses, speed, as the domain on which a continuous probability distribution is defined. In the case of qualitatively different responses, each response class may have positive probability for its occurrence, whereas with continuous probability distribution Logan's dependent variable is probability of certain class of response strengths, probability that behavior with non-zero interval of quantitative properties will occur. Because, with continuous probability distribution probability of countably many response strengths is zero.

Dealing with the classical conditioning 
situation, the acquisition process in terms of the probability of occurrence of CR was deduced by Estes (1950) from a set of postulates without the intervention of generalized concept of response. According to his conceptualization, there is associated with an organism a stimulus population $\mathrm{S}$ and a response class $R$. Each element in $\mathrm{S}$ is connected to only one element in $\mathrm{R}$. By defining a probability measure on $\mathrm{S}$ according to a function which associates to each element in $\mathrm{S}$ an element in $\mathrm{R}$, the modification of behavior will be nothing but a change in this function.

However, Estes left an exercise for the student of psychology. Because in his formalization, the concept of stimulus is extremely inaccessible. It may be argued that the sampling process does apply to the cell assembly corresponding to US rather than to the stimulus population controlled by CS. The sampling ratio will then take the meaning of the relative amount of neuronal firing in nonspecific projection system. The neuronal firing due to nonspecific source is assumed to provide a general facilitative background and hence to influence the threshold of specific cell assemblies.

A recent study indicates that in the instrumental conditioning the error and latency measures can be deduced rigorously from the probability distribution of correct and incorrect response classes with certain experimental conditions (Liu, 1961). Major confusion at the present stage of psychology seems to arise from lack of analyzing experimental conditions properly.

\section{Nature of Experimental Setup}

The classical conditioning is characterized by several features. Imposing an experimental restricton for insuring the occurrence of response is done by applying US. The major aim in the study of the classical conditioning is to see change in the response occurrence subsequent to the removal of this experi- mental restriction, US. To attain this purpose, additional feature is added to the main experimental restriction, US. and the functional transfer of the effect of the main restriction is studied by its omission. The additional feature in the classical conditioning is the administration of CS. When the additional feature is absent, the experimental restriction may be changed slightly, and its effect on the response occurrence is studied. The latter paradigm is usually referred to as generalization experiment.

Therefore, we distinguish several experimental features essential to the description of the classical conditioning and generalization situations. First, there is the original restriction which insures the occurrence of certain measurable aspect of behavior. Second, there is modified restriction under which behavior modifications are to be investgated. Third, to obtain this modified restriction there is an intermediate process for establishing modified restriction. In the classical conditioning the intermediate process is usually known by the name of acquisition. In generalization experiments, the intermediate process for the establishment of the modified restriction is not necessary, for the modified restriction in this case is very similar to the original restriction.

The learning situations mentioned above have one essential characteristic in common. A new experimental situation is constructed so that the testing situation is different from the original situation. In another class of learning situations, the situation itself remains constant while certain aspect of behavior undergoes change as a consequence of differential behavioral outcomes. The latter class of learning situations is ordinarily understood as typical of instrumental conditioning situation. All the following analysis is restricted to the calss of instrumental conditioning.

The essential feature of experimental setup is to provide a situation under which behavior modification takes place con- 
comitant with a certain set of experimental operations. A behavior modification exists when the present behavior is different from the previous. In other words the occurrrence of behavior is prerequisite for the demonstration of a reaction change.

A set of behavior tendencies of an organism is so complicated that it is impractical to consider all of them at the same time. Therefore an experimental setup is devised in such a way that only a few of them appear in this controlled situation. Runway situation is an experimental setup where only one type of response, running, takes place. The occurrence of the other responses may be possible. When this happens, however, the experimental control is considered not satisfactory. There are some situations providing the possibility of occurrence of two response classes. For example, in a Skinner box the occurrence of bar pressing may be considered constituting the first response class with the rest included in the second response class.

When an experimental restriction is such that only one response class is a probable outcome, the possible behavior modification is in its amplitude or speed. Under different experimental restriction with a collection of mutually exclusive response classes as a possible outcome, behavior modification takes place in the probability distribution of response tendencies. This probability distribution is usually measured in terms of frequency.

Thus the nature of the probability distribution of response classes imposed by a particular experimental setup determines invariably the types of behavioral measures for recording behavior modifications. The major theme of this section is to assert that the initial probability distribution of response classes and drive shift function characterize entirely that particular experimental setup. For this purpose, the concept of drive shift function will be clarified in the following.
Let the initial probability distribution of $n$ mutually exclusive response classes be such that each response class has positive probability with $n$ greater than or equal to two. When some response classes have zero probability at the beginning of an experiment, those response classes will be eliminated from consideration. The drive shift function is defined on a set of these response classes. To take an example, consider a Skinner box with a horizontal and a vertical bars and suppose that the temperature within the Skinner box is very low. Let a pretraining be given such that the rat in this situation presses the horizontal bar with probability $p_{1}$ and the vertical bar with probability $p_{2}$. When the horizontal and vertical bars are pressed, the temperature in the Skinner box is supposed to rise to $t_{1}$ and $t_{2}$ respectively. This means that to each response class there is associated a definite amount of drive shift produced by the temperature change. This correspondence of response class and temperature change is the drive shift function. The range of the drive shift function may be very simple, such as consisting of only two values, a fixed amount of drive shift and no shift at all. Ordinary instrumental conditioning is characterized by this special drive shift function. When the domain of the drive shift function is fairly large, the function can be made arbitrarily smooth. The extinction setup in the instrumental conditioning is obtained by assigning to each response class zero drive shift. This type of drive shift function is the simplest one.

In the case of discrimination learning a response contingent upon a distinctive stimulus which is experimentally imposed will be regarded as constituting a distinctive response class. Thus the right and left turning responses with two distinctive stimuli, one positive and the other negative, generate four distinctive response classes. To these response classes a probability distribution is attached. Simi- 
larly the drive shift function used to define discrimination learning is very special one viewing from its general definition. From mathematical point of view the drive shift function in this case is actually defined on a product set of stimulus and response. However, the stimulus situation, on the other hand, can be conveniently regarded as an experimental restriction for defining response classes.

\section{Law of Maximum Drive Shift}

The following analysis is restricted to the case of discrete probability distribution. Let the initial drive intensity before the occurrence of response be denoted by $d_{0}$ and the changed drive intensity concomitant with the occurrence of the response class $R_{i}$ be indicated by $d_{i}$. To get a useful drive shift function it is reasonable to take their ratio. Since in this way the particular unit associated with drive intensity can be disregarded. If there is no drive shift, it is plausible to have the functional value equal to zero. When drive intensity shifts in the other direction, the different sign for the functional value is desirable. For these together with some other reasons, the drive shift function is defined to be $\log d_{0} / d_{i}$.

To avoid the case of $d_{i}=0$, which leads to a meaningless expression, a constant $\delta$ is assumed to be added to $d_{i}$ to give net drive intensity level after the occurrence of the response class $R_{i}$. The existence of this constant small in quantity is justified empirically by the slightly agitated state of organism after response evocation. Therefore, the expectation of the drive shift function can be written in the following way.

$$
\sum_{i=1}^{n} P\left(A_{i}\right) \log d_{0} / d_{i}+\delta,
$$

where $A_{i}$ represents an event connected with the occurrence of the response class $R_{i}$, and $P\left(A_{i}\right)$ is the probability of occurrence of $R_{i}$. The maximum of the expectation of the drive shift function is attained at the minimum $d_{i}$ with the probability of $A_{i}$ equal to one. In other words, the expected value of the drive shift function is the greatest when animal chooses a response which produces the least drive intensity with probability one. To prove this statement, it will be first noticed that the expectation of the drive shift function can be decomposed into two simple parts.

$$
\begin{gathered}
\sum_{i=1}^{n} P\left(A_{i}\right) \log d_{0} / d_{i}+\hat{o}=\sum_{i=1} P\left(A_{i}\right) \log d_{0} \\
-\sum_{i=1}^{n} P\left(A_{i}\right) \log \left(d_{i}+\delta\right) \\
=\log d_{0}-\sum_{i=1}^{n} P\left(A_{i}\right) \log \left(d_{i}+o\right) .
\end{gathered}
$$

Hence, the proof of the maximum value of the expectation of the drive shift function is equivalent to proving the minimum of $\sum_{i=1}^{n} P\left(A_{i}\right) \log \left(d_{i}+\delta\right)$ at the same point, if $d_{i}+\delta \leq d_{0}$ for all $i$. The latter condition is understood as providing the case of nonpunishing situation.

Let $d_{i_{0}}$ be the minimum of $d_{i}$ for all $i$. Then it is asserted that

$$
\log \left(d_{i_{0}}+\hat{o}\right) \leq \sum_{i=1}^{n} P\left(A_{i}\right) \log \left(d_{i}+\tilde{o}\right)
$$

This inequality is equivalent to

$$
\begin{gathered}
\sum_{i \neq i_{0}} P\left(A_{i}\right) \log \left(d_{i}+\delta\right) \\
-\left[1-P\left(A_{i_{0}}\right)\right] \log \left(d_{i_{0}}+\delta\right) \geq 0
\end{gathered}
$$

Since $d_{i_{0}}$ is the minimum of $d_{i}$ for all $i$,

$$
\begin{aligned}
\sum_{i \neq i_{0}} P\left(A_{i}\right) \log \left(d_{i}+\delta\right) & \geq \sum_{i \neq i_{0}} P\left(A_{i}\right) \log \left(d_{i_{0}}+\hat{o}\right) \\
& =\left[1-P\left(A_{i_{0}}\right)\right] \log \left(d_{i_{0}}+\tilde{o}\right)
\end{aligned}
$$

Substituting this inequality into [2], [1] is proved.

Thus, the asymptotic property of behavior change is stated in terms of the probability distribution by means of the corresponding property of the drive shift function in a rigorous way. The expectation of the drive shift function looks like a function of two variables. Actually it is a function of one variable, since $P\left(A_{i}\right)$ $=P\left(d_{i}\right)$. The last part of the decomposed expectation of the drive shift function 
$\sum_{i=1}^{n} P\left(A_{i}\right) \log \left(d_{i}+\hat{o}\right)$ is very similar in from to the entropy in information theory.

\section{SUMMARY}

Since response occurrence is experimentally imposed, any experimental setup in the instrumental conditioning can be characterized entirely by the probability distribution of response classes and the drive shift function defined on these response classes. The nature of the probability distribution invariably sets up a continuum of behavioral measure along which behavior change takes place. As a consequence, the law of maximum reward can be stated in terms of the asymptotic nature of the probability distribution of response classes.

\section{REFERENCES}

EsTES, W. K. Toward a statistical theory of learning. Psychol. Rev., 1950, 57, 94-107.

Hull, C. L. Principles of behavior. New York: Appleton-Century, 1943.

LiU, I. A derivation of latency equation. Bull. Math. Biophysics, 1961, Sep. issue.

LOGAN, F. A. A micromolar approach to behavior theory. Psychol. Rev., 1956, 63, 63-73.

SPENCE, K.W. Behavior theory and conditioning. New Haven: Yale Univ. Press, 1956.

(Received Oct. 11, 1961) 